\title{
Modern trends in the management of head and neck paragangliomas
}

\author{
Carlos Suárez $^{1,2,3}$ • Verónica Fernández-Alvarez ${ }^{3,4}$ - Hartmut P. H. Neumann ${ }^{5}$. \\ Carsten C. Boedeker ${ }^{6} \cdot$ Christian Offergeld $^{7} \cdot$ Alessandra Rinaldo $^{8}$. \\ Primož Strojan' ${ }^{9}$ Alfio Ferlito ${ }^{10}$
}

Received: 17 September 2015/ Accepted: 2 October 2015/Published online: 16 October 2015

(C) Springer-Verlag Berlin Heidelberg 2015

Head and neck paragangliomas (HNPG) are rare, mostly benign neoplasms that usually exhibit an indolent growth pattern although they can be associated with compression and infiltration of adjacent cranial nerves and, depending on the site of origin, also bone and intracranial structures. Less than $5 \%$ of the tumors are considered malignant based on the presence of metastases and not local invasion. Carotid body tumors accounts for two-thirds of HNPG, whereas vagal paragangliomas are showing the highest tendency toward malignant character.

This article was written by members and invitees of the International Head and Neck Scientific Group (www.IHNSG.com).

Alfio Ferlito

a.ferlito@uniud.it

1 Fundación de Investigación e Innovación en Biomedicina de Asturias, Oviedo, Spain

2 Instituto Universitario de Oncología del Principado de Asturias, Oviedo, Spain

3 Universidad de Oviedo, Oviedo, Spain

4 Department of Vascular Surgery, Hospital de Cabueñes, Gijón, Spain

5 Department of Nephrology and Hypertension, AlbertLudwigs-University, Freiburg, Germany

6 Department of Otorhinolaryngology-Head and Neck Surgery, HELIOS Hanseklinikum Stralsund, Stralsund, Germany

7 Department of Otorhinolaryngology-Head and Neck Surgery, University of Freiburg, Freiburg, Germany

8 University of Udine School of Medicine, Udine, Italy

9 Department of Radiation Oncology, Institute of Oncology, Ljubljana, Slovenia

10 Coordinator of the International Head and Neck Scientific Group, Padua, Italy
Despite the usual treatment of benign tumors is surgery, the risks of the treatment-related complications and potential deterioration of quality of patient's live, however, should not be greater than the risk brought by the tumor in its natural course. Watchful waiting and radiotherapy are widely accepted in the management of vestibular schwannomas, a tumor that is usually indolent but, like HNPG, also has an unpredictable growth pattern. Review of different national tumor registry databases revealed that in the United States there has been a significant shift in management of vestibular schwannomas over a decade, with increasing tendency toward observation and radiotherapy, whereas the proportion of operated cases declined to near a half of the total [1-3]. Similar studies on the trends of treatment are lacking in HNPG. Systematic analysis of the literature has shown that most of the HNPG have been treated surgically, with no data on the impact of observation in the management of these tumors $[4,5]$.

\section{"Wait and see" policy}

Likewise, due to prevailingly indolent nature HNPG with low growth potential the decision on optimal treatment in HNPG is delicate, even more in view of the facts that tumor growth in individual paraganglioma (PG) case cannot be predicted and mortality caused directly by the tumor is a rare event that occurs in only $1-4 \%$ of patients [4]. According to Jansen et al. [6], the median increase in size in a series of $48 \mathrm{HNPG}$ was $0.83 \mathrm{~mm} / \mathrm{year}$. A volume increase of $20 \%$ was noted in $60 \%$ of the tumors, with a median increase in dimension in this subgroup of $1 \mathrm{~mm} /$ year. In addition, tumor doubling time has been universally estimated as low, ranging between 4.2 and 13.8 years for HNPG [7, 8]. 
Therefore, a "wait and see" policy should be considered as one of the treatment strategies for these tumors, similarly to other intra/extracranial benign tumors, such as vestibular schwannomas, meningiomas, etc. This assumption is supported by a study carried out by van der Mey et al. [9] in 175 HNPG. The results of radical surgical treatment in this series were disappointing in terms of the rate of complete resections achieved and increased morbidity for jugular paragangliomas (JPG). During the follow-up period (mean 13.5 years), none of the patients died of residual or recurrent tumor, irrespective of the mode (wait and see, surgery, and radiotherapy) or outcome of treatment. These results raise the question of whether this natural behavior is really improved by intervention.

In addition, Rodriguez-Cuevas et al. [10] followed 41 patients with carotid body tumors (CBT) who did not receive any treatment after the diagnosis was established. The mean size of these tumors was $5.3 \mathrm{~cm}$, and during a median follow-up of 47 months, no patient reported additional symptoms, accelerated enlargement of the tumor, or metastasis. Recently, Langerman et al. [11] reported the results of observation in a group of 47 cervical PG [28 CBT and 19 vagal paragangliomas (VPG)]. During a mean follow-up of 5 years, 19 tumors remained stable in size (42\%), 17 grew (38\%), and 9 regressed (20\%). The mean enlargement of the tumors that showed any sign of growth was $0.2 \mathrm{~cm} /$ year.

Taken into account the biological behavior of HNPG, a "wait and see" policy should be considered as one of the valid treatment strategies for these tumors. Using this approach, the main goal in JPG and VPG is to reduce morbidity rather than attempting to increase survival. However, it can be argued that a wait and see policy could increase the risk for development of new cranial nerve deficits. Nevertheless, only $7.5 \%$ of patients with VPG in whom no active treatment was implemented, developed cranial nerve palsy during an average follow-up of 8.5 years [12].

Occasionally cranial nerve involvement may become clinically apparent in HNPG, even without radiologically detectable growth of the tumor. Taking into account that small tumors have potential to be resected with less morbidity, early surgery can be chosen when the goal is to eradicate the tumor and prevent future morbidity. However, the main argument against surgical intervention is the appreciable number of incomplete resections and, more importantly, not negligible risk of morbidity, even in low-volume lesions.

\section{Role of the radiotherapy}

Radiotherapy has been criticized for the high exposure of normal neural tissue and bone to radiation, resulting in considerable risk of radiation-induced complications.
However, most of the severe complications recorded after radiotherapy of HNPG occurred in patients who received 55-65 Gy. On the other hand, there is no evidence that doses higher than $45 \mathrm{~Gy}$ improve probability of tumor control, whereas the risk of significant complications after delivering a dose of $45 \mathrm{~Gy}$ is negligible. Stereotactic radiosurgery (SRS) and stereotactic radiotherapy seem to play an increasingly important role in the treatment of these tumors due to higher capability of sparing adjacent normal tissues than more conventional radiotherapy techniques. Nevertheless, the reported series are based on small numbers of patients with shorter follow-up than external beam radiotherapy (EBRT) and surgical series.

\section{Treatment of jugular and vagal paragangliomas}

The results of surgery, EBRT, and SRS have been analyzed in a systematic review including 2042 patients with JPG or VPG [4]. Tumor control failure rate, major complication rate, and the number of cranial nerve palsies after treatment were significantly higher in surgical than in radiotherapytreated patients. The results of SRS and EBRT in JPG were also compared and no significant differences were observed in tumor control. Among SRS series, a $93.7 \%$ rate of local control was observed, compared to 89.1 and $78.2 \%$ obtained with EBRT and surgery, respectively. No deaths were associated with SRS and the mortality rates secondary to surgery and EBRT were 1.6 and $2 \%$, respectively. With surgery, cranial nerve palsies increased to $181.6 \%$ in comparison to preoperative levels, whereas with SRS and EBRT cranial nerve palsies decreased for 8.8 and $4.1 \%$, respectively. A similar situation was observed related to hearing loss: most patients in the surgical group developed a deep sensorineural or conductive hearing loss, whereas only $6.5 \%$ of patients treated with SRS had permanent worsening. Concerning VPG, results of surgery were even worse in terms of cranial nerve preservation: only $4.9 \%$ of patients retained function of the vagus nerve after surgical excision.

For every HNPG, except tympanic paragangliomas (TPG), surgery will not directly improve the clinical situation of the patient. In order to be selected for treatment with surgery or radiotherapy rather than for observation, tumors should be symptomatic or show signs of progression on radiologic studies, which is defined as an increase in volume of $>20 \%$ in 1 year. TPG are usually small and generally have a sporadic origin, presenting as isolated lesion in the middle ear cleft. Its resection may be carried out with a low risk of morbidity and with the advantage of suppressing both the pulsatile tinnitus and the hearing loss.

Surgery of VPG almost invariably leads to vagal nerve palsy and should be reserved for those patients who already 
have a palsy, and even then, radiotherapy and observation are worth considering because additional cranial nerve palsies can occur.

For JPG, it seems more adequate to use surgery for small tumors with low risk of damaging hearing and cranial nerves and to treat the rest with radiotherapy. In these cases, the ability to preserve the lower cranial nerves is directly proportional to the size of the tumor. Tumor size also determines success in hearing preservation. Therefore, in Fisch Class C1-2 tumors the "fallopian bridge" technique can be used, which allows the preservation of the external auditory canal and tympanic membrane, and the facial nerve is left within its bony canal [13, 14]. Nevertheless, a conservative policy based on "wait and scan", can also be adopted in these tumors.

To reduce pulsatile tinnitus and preserving or improving residual hearing in JPG of patients of advanced age, the use of a limited middle ear/mastoid tumor resection and postoperative SRS to tumor remnants in the jugular foramen region has been advocated [15].

Other strong indications for surgery are patients with facial nerve palsy suitable for repair with a cable graft, tumors that increase in size after radiotherapy, or tumors that have significant intracranial extension and are life threatening due to mass effect. After a subtotal resection, SRS is a safe and effective option for treatment of residual tumor. Tumors too large for radiosurgery can be managed with EBRT.

\section{Treatment of carotid body paragangliomas}

Traditionally, management of CBT includes surgery as main option assuming a low risk of postoperative complications. Clinical stage of CBT is usually established according to Shamblin classification. Shamblin Class I CBT are localized tumors with splaying of the carotid bifurcation, but little attachment to the carotid vessels. Shamblin Class II CBT partially surround the carotid vessels. Shamblin Class III CBT intimately surround the carotid.

Nevertheless, Shamblin Class III tumors are strongly associated with postoperative neurological damage compared to tumors staged as Class I/II [16]. According to Makeieff et al. [17], the rate of serious complications (i.e., permanent nerve palsy, vascular complications) was $2.3 \%$ for Shamblin Class I/II tumors and $35.7 \%$ for Shamblin Class III tumors. Nevertheless, internal carotid resection with or without vascular reconstruction when the artery is enveloped by the tumor has been reported in as much as $12.5-18 \%$ of surgically treated patients [5, 18, 19]. According to Vogel et al. [19] who carried out a systematic review including 1988 patients who underwent CBT surgery, those with reconstruction of the carotid artery had significantly greater rate of mortality (1.61 vs. $0.59 \%)$ and stroke (17.7 vs. $3.5 \%$ ) compared to the rest of patients. To summarize, although "wait and scan" is an option in most of the patients younger than 60 years with a single Class I CBT, surgery should be considered as the first choice therapy, since under these circumstances it produces minimal morbidity, almost no mortality, and a very good chance to really cure the patient.

Several reports have been published about the use of radiotherapy as first choice of treatment for CBT. Its main advantages are the avoidance of immediate major complications and the high rates of local control, usually manifested only as cessation of growth or partial regression. However, a consistent relationship between neck radiotherapy and carotid stenosis has been demonstrated in the literature, with an increase of $12 \%$ in the stroke risk within 15 years following radiotherapy [20]. Nevertheless, to date no such case has been reported in CBT patients after radiotherapy, probably because doses of radiotherapy in these patients are much lower compared to doses used in head and neck squamous cell carcinomas. Another factor to consider is the risk of radiation-induced sarcoma development. The risk is probably $1 \%$ or less at 10 years following radiotherapy, but continues to gradually increase over time. Late radiation-related meningiomas have also been described after treatment [21].

Treatment outcomes of surgery and EBRT were analyzed in a systematic review including 2,302 patients with CBT [5]. Long-term control of the disease was obtained in $93.8 \%$ of the patients who received surgical treatment, and in $94.5 \%$ of patients treated with EBRT. The common/ internal carotid artery was resected in 271 patients with immediate reconstruction in 212 patients. As a consequence, $3 \%$ of the patients experienced a permanent stroke. It is important to mention that 26 patients $(1.3 \%)$ died due to postoperative complications. No deaths were associated with EBRT, but the most important difference between surgery and EBRT was the rate of morbidity. With surgery, cranial nerve palsies increased 3.9-fold \% in comparison to preoperative levels, while with EBRT cranial nerve palsies remained unaffected.

\section{Infrequent locations of HNPG}

Thyroid paraganglioma is an extremely rare tumor and frequently mistaken for other thyroid hypervascular neoplasms. To date, all the cases reported in the literature have demonstrated a benign course, but on intraoperative frozen section biopsy very often are misdiagnosed as carcinomas, due to the extension into adjacent thyroid tissues and vascular invasion. Notably, approximately $80 \%$ of thyroid 
paragangliomas are associated with germline variants, with implications for additional tumors and a potential risk for the family. Increased awareness of its potential presentation in thyroid and its characteristic features is essential for avoiding diagnostic and therapeutic pitfalls.

Laryngeal paragangliomas are rare submucosal lesions that are mainly located in the supraglottic larynx. Symptoms arise when the lesions become large enough to impair function. Image studies can permit characterization of the vascularity of the lesion, avoiding the need for biopsy. Treatment should always comprise open functional surgery or laser excision, which are preferable to radiation.

Sinonasal paragangliomas are frequently malignant especially for patients with history of genetic mutations such as SDHB mutations. If malignant, they are very aggressive, with rapid local spread as well as high metastatic potential despite surgical resection. Extension to the skull base may preclude complete surgical resection. In these cases, (131)iodine-MIBG can be used as adjuvant therapy in postoperative external beam radiation.

\section{Genetic background of HNPG}

Different studies have demonstrated that approximately one-third of HNPG are caused by a germline mutation in $\mathrm{SDH}$ genes. Mutations of the SDHD gene are the most commonly found. Patients with SDHD gene mutations are at high risk for the development of HNPG (91-98\%) with multiple lesions as a key feature of this syndrome which can be found in the majority of patients (60-79\%) [22]. Multiple tumors, including VPG and JPG, should be managed conservatively with watchful waiting and radiotherapy in the case of growth of the tumors, particularly if bilateral, due to the devastating effects of bilateral vagus nerve palsies. When bilateral CBT are found, an accepted strategy is to resect the smaller tumor first because this can usually be done with minimal or only temporary risk of cranial nerve injury. Resection of the second tumor is controversial, even if its size is small, because bilateral carotid sinus denervation may often result in acute baroreflex failure, producing severe, labile hypertension, headache, diaphoresis, and emotional instability [23]. Alternatively, one or both CBT may be irradiated with little risk of severe complications.

Malignant PG are uncommon, representing around $5 \%$ of all HNPG. It is usually impossible to differentiate between benign and malignant PG on the basis of histology, the diagnosis being almost always made by detecting nodal or distant metastases. Approximately, two-thirds of malignant tumors occur in SDHB mutation carriers.
Surgery of malignant HNPG is the preferred treatment in order to avoid further dissemination of the disease.

The prevalence of malignant paragangliomas in patients with SDHB mutation with manifest disease ranges from 0 to $54 \%$. van Hulsteijn et al. [24] performed a meta-analysis on the risk of developing malignant $\mathrm{PG}$ in SDH mutation carriers and observed that in studies comprising only mutation carriers with manifest disease, the pooled prevalence of malignant tumors was $23 \%$ for SDHB mutation. This fact set the dilemma of what to do if asymptomatic HNPG in SDHB carriers are detected. The risks of a surgical treatment must be weighed against the frequently indolent natural course of the disease, thus decision on treatment should be highly individual and in agreement with well-informed patient.

Secreting PG accounts for only less than $5 \%$ of all HNPG [25]. Within the cytoplasm of the chief cells are secretory granules containing catecholamines. Persistent chief cells have been found after radiotherapy, and this may explain why catecholamine secretion persists after radiotherapy. Thus, the management of patients with secreting HNPG includes surgery as the preferred option, particularly if the elevated catecholamine levels are accompanied by symptoms.

Finally, a comprehensive flowchart for decision making is shown in Fig. 1.

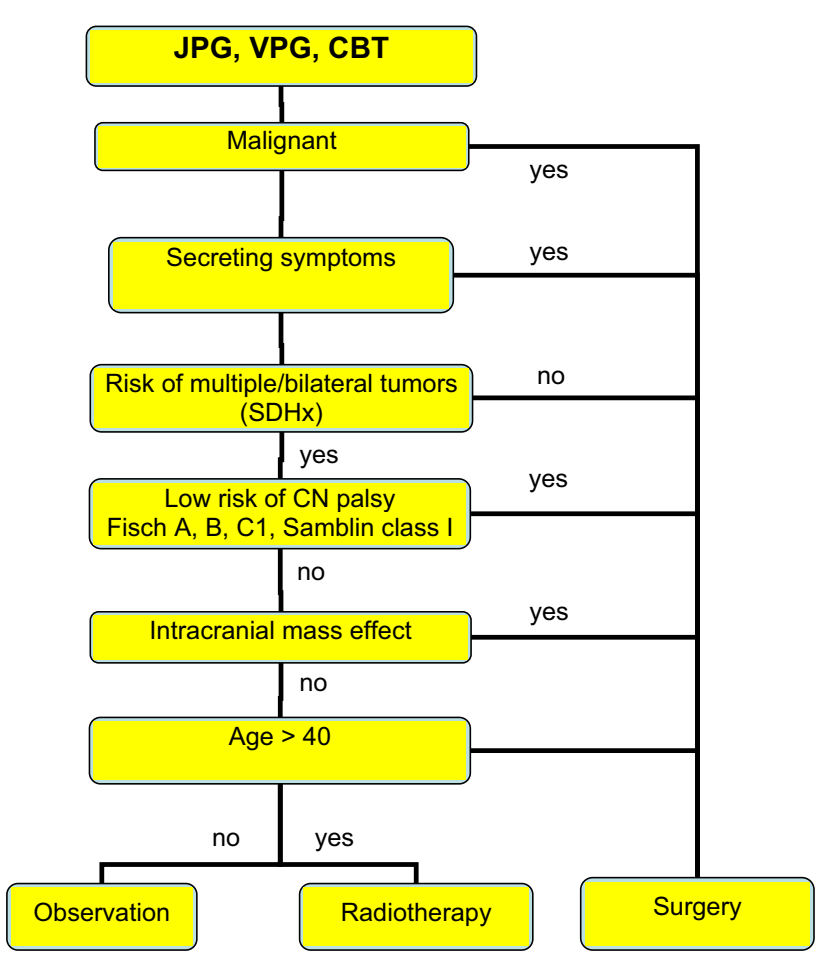

Fig. 1 Algorithm for management of HNPG 


\section{References}

1. Patel J, Vasan R, van Loveren H, Downes K, Agazzi S (2014) The changing face of acoustic neuroma management in the USA: analysis of the 1998 and 2008 patient surveys from the acoustic neuroma association. Br J Neurosurg 28:20-24. doi:10.3109/ 02688697.2013.815323

2. Gal TJ, Shinn J, Huang B (2010) Current epidemiology and management trends in acoustic neuroma. Otolaryngol Head Neck Surg 142:677-681. doi:10.1016/j.otohns.2010.01.037

3. Babu R, Sharma R, Bagley JH, Hatef J, Friedman AH, Adamson C (2013) Vestibular schwannomas in the modern era: epidemiology, treatment trends, and disparities in management. J Neurosurg 119:121-130. doi:10.3171/2013.1.JNS121370

4. Suárez C, Rodrigo JP, Bödeker CC, Llorente JL, Silver CE, Jansen JC, Takes RP, Strojan P, Pellitteri PK, Rinaldo A, Mendenhall WM, Ferlito A (2013) Jugular and vagal paragangliomas: a systematic study on management with surgery and radiotherapy. Head Neck 35:1195-1204. doi:10.1002/hed.22976

5. Suárez C, Rodrigo JP, Mendenhall WM, Hamoir M, Silver CE, Grégoire V, Strojan P, Neumann HPH, Obholzer R, Offergeld C, Langendijk JA, Rinaldo A, Ferlito A (2014) Carotid body paragangliomas: a systematic study on management with surgery and radiotherapy. Eur Arch Otorhinolaryngol 271:23-34. doi:10. 1007/s00405-013-2384-5

6. Jansen JC, van den Berg R, Kuiper A, van der Mey AG, Zwinderman AH, Cornelisse CJ (2000) Estimation of growth rate in patients with head and neck paragangliomas influences the treatment proposal. Cancer 88:2811-2816

7. Persky MS, Setton A, Niimi Y, Hartman J, Frank D, Berenstein A (2002) Combined endovascular and surgical treatment of head and neck paragangliomas-a team approach. Head Neck 24:423-431

8. Foote RL, Pollock BE, Gorman DA, Schomberg PJ, Stafford SL, Link MJ, Kline RW, Strome SE, Kasperbauer JL, Olsen KD (2002) Glomus jugulare tumor: tumor control and complications after stereotactic radiosurgery. Head Neck 24:332-338

9. van der Mey AG, Frijns JH, Cornelisse CJ, Brons EN, van Dulken H, Terpstra HL, Schmidt PH (1992) Does intervention improve the natural course of glomus tumors? A series of 108 patients seen in a 32-year period. Ann Otol Rhinol Laryngol 101:635-642

10. Rodríguez-Cuevas S, López-Garza J, Labastida-Almendaro S (1998) Carotid body tumors in inhabitants of altitudes higher than 2000 meters above sea level. Head Neck 20:374-378

11. Langerman A, Athavale SM, Rangarajan SV, Sinard RJ, Netterville JL (2012) Natural history of cervical paragangliomas: outcomes of observation of 43 patients. Arch Otolaryngol Head Neck Surg 138:341-345. doi:10.1001/archoto.2012.37
12. Bradshaw JW, Jansen JC (2005) Management of vagal paraganglioma: is operative resection really the best option? Surgery 137:225-228

13. Suárez C, Sevilla MA, Llorente JL (2007) Temporal paragangliomas. Eur Arch Otorhinolaryngol 264:719-731

14. Llorente JL, Obeso S, López F, Rial JC, Coca A, Suárez C (2014) Comparative results of infratemporal fossa approach with or without facial nerve rerouting in jugular fossa tumors. Eur Arch Otorhinolaryngol 271:809-815. doi:10.1007/s00405-013-2642-6

15. Cosetti M, Linstrom C, Alexiades G, Tessema B, Parisier S (2008) Glomus tumors in patients of advanced age: a conservative approach. Laryngoscope 118:270-274

16. Plukker JT, Brongers EP, Vermey A, Krikke A, van den Dungen JJ (2001) Outcome of surgical treatment for carotid body paraganglioma. Br J Surg 88:1382-1386

17. Makeieff M, Raingeard I, Alric P, Bonafe A, Guerrier B, MartyAne C (2008) Surgical management of carotid body tumors. Ann Surg Oncol 15:2180-2186. doi:10.1245/s10434-008-9977-z

18. Anand VK, Alemar GO, Sanders TS (1995) Management of the internal carotid artery during carotid body tumor surgery. Laryngoscope 105:231-235

19. Vogel TR, Mousa AY, Dombrovskiy VY, Haser PB, Graham AM (2009) Carotid body tumor surgery: management and outcomes in the nation. Vasc Endovasc Surg 43:457-461. doi:10.1177/ 1538574409335274

20. Haynes JC, Machtay M, Weber RS, Weinstein GS, Chalian AA, Rosenthal DI (2002) Relative risk of stroke in head and neck carcinoma patients treated with external cervical irradiation. Laryngoscope 112:1883-1887

21. Dupin C, Lang P, Dessard-Diana B, Simon JM, Cuenca X, Mazeron JJ, Feuvret L (2014) Treatment of head and neck paragangliomas with external beam radiation therapy. Int J Radiat Oncol Biol Phys 89:353-359. doi:10.1016/j.ijrobp.2014.02.010

22. Boedeker CC, Hensen EF, Neumann HPH, Maier W, van Nederveen FH, Suárez C, Kunst HP, Rodrigo JP, Takes RP, Pellitteri PK, Rinaldo A, Ferlito A (2014) Genetics of hereditary head and neck paragangliomas. Head Neck 36:907-916. doi:10.1002/hed. 23436

23. Robertson D, Hollister AS, Biaggioni I, Netterville JL, Mosqueda-Garcia R, Robertson RM (1993) The diagnosis and treatment of baroreflex failure. N Engl J Med 329:1449-1455

24. van Hulsteijn LT, Dekkers OM, Hes FJ, Smit JW, Corssmit EP (2012) Risk of malignant paraganglioma in SDHB-mutation and SDHD-mutation carriers: a systematic review and meta-analysis. J Med Genet 49:768-776. doi:10.1136/jmedgenet-2012-101192

25. Erickson D, Kudva YC, Ebersold MJ, Thompson GB, Grant CS, van Heerden JA, Young WF Jr (2001) Benign paragangliomas: clinical presentation and treatment outcomes in 236 patients. J Clin Endocrinol Metab 86:5210-5216 